\title{
Cribado y transmisión congénita de la enfermedad de Chagas en población usuaria del Hospital Dr. Félix Bulnes Cerda y Atención Primaria de Salud del Servicio de Salud Metropolitano Occidente de Santiago, Chile
}

\author{
Screening and congenital transmission of Chagas Disease in the user population \\ of the Dr. Félix Bulnes Cerda Hospital and Primary Health Care Services \\ of the Metropolitan Health Service West of Santiago, Chile
}

\author{
Marisol Denegri C. ${ }^{2}$, Alan Oyarce F. ${ }^{1}$, Paula Larraguibel C. ${ }^{3}$, Iris Ramírez V. ${ }^{3}$, Elizabeth Rivas B. ${ }^{3}$, \\ Gisella Arellano P. ${ }^{3}$, Andrea Báez M. ${ }^{4}$ y Nancy P. Maulén L. ${ }^{3}$
}

'Sección de Parasitología, Instituto de Salud Pública de Chile.
2Policlínico Chagas, Hospital Dr. Félix Bulnes Cerda.
${ }^{3}$ Laboratorio Clínico Central, Hospital Dr. Félix Bulnes Cerda. Santiago, Chile.
${ }^{4}$ Instituto de Estadística, Universidad Austral de Chile. Valdivia, Chile.

Financiamiento: Programa de Control y Prevención de la Enfermedad de Chagas del Ministerio de Salud de Chile y Hospital Dr. Félix Bulnes Cerda del Servicio de Salud Metropolitano Occidente.

Los autores declaran la ausencia de conflictos de interés.

Recibido: 8 de septiembre de 2019 / Aceptado: 30 de marzo de 2020.

\section{Resumen}

Introducción: El año 2016, el Hospital Dr. Félix Bulnes Cerda (HDFBC) implementó el tamizaje obligatorio de anticuerpos anti Trypanosoma cruzi, para pesquisar nuevos casos de la enfermedad de Chagas $(\mathrm{ECH})$ en mujeres embarazadas y recién nacidos $(\mathrm{RN})$ y brindar tratamiento antiparasitario oportuno. Ello, porque la transmisión transplacentaria de T. cruzi continúa siendo un importante problema de salud pública. Objetivo: Presentar la información epidemiológica generada luego de la implementación del cribado de la ECH en el HDFBC del SSMOCC, al cabo de dos años de iniciada su operación. Material y Métodos: Cribado por inmunoquimioluminiscencia (IQLU), confirmación por IFI, ELISA y/o western blot. Seguimiento de RN: mediante RPC, IFI, ELISA e IQLU. Resultados: Entre abril de 2016 y julio de 2018 se analizó un total de 14.012 muestras de pacientes; 62 resultaron reactivas $(0,53 \%$ seropositividad) y 28 fueron confirmadas positivas (prevalencia de $0,2 \%$ ). Del total, 11.780 fueron de mujeres gestantes ( $\mathrm{n}$ : 41 casos reactivos y 10 confirmadas con $\mathrm{ECH}$ ), con prevalencia de $0,085 \%$. Los restantes 18 casos positivos fueron usuarios no gestantes, con sospecha clínica de ECH y casos de estudios familiares, mostrando una prevalencia de $0,81 \%$. El seguimiento a $10 \mathrm{RN}$ y lactantes hijos de madres chagásicas descartó la transmisión transplacentaria Completaron tratamiento con nifurtimox

\begin{abstract}
Background: In 2016, the Hospital Dr. Félix Bulnes Cerda (HDFBC) implemented the mandatory screening of anti Trypanosoma cruzi antibodies in pregnant women, thus complying with national regulations to detect new Chagas disease cases (CHD) in mother and child, whose early detection mediates timely pharmacological treatment. This, because the congenital transmission continues the main active transmission mechanisms of $T$. cruzi and a major public health problem for the country. Aim: To present the epidemiological information generated after the implementation of the ECH screening in the SSFCC HFBC, two years after its operation began. Methods: Screening test: inmunequimioluminiscence (IQLU), certification by IFI, ELISA and/or western blot. Follow up of newborn infants with PCR, IFI, ELISA and IQLU. Results: Between April 2016 and July 2018, 14.012 subject's samples were examined of which 62 resulted reactive $(0.53 \%$ seropositivity) in the total studied population where 28 patients resulted positive for CHD, which corresponds to a $0.2 \%$ prevalence. Of the total population, 11.780 were pregnant women, of these 41 were reactive and 10 were confirmed with CHD thus showing a prevalence of $0.085 \%$. The other 18 positive cases of non-pregnant patients corresponded a prevalence of $0.81 \%$. Also, 10 newborn and infant children of mothers with CHD were followed
\end{abstract}


$30 \%$ de las madres. Discusión: La implementación del programa en el HDFBC cumple los objetivos de cribado, pesquisa y diagnóstico de la ECH así como el seguimiento y tratamiento oportuno del RN. La prevalencia de $0,085 \%$ de la ECH en la población gestante como la de $0,2 \%$ en población total son significativamente menores ( $\mathrm{p}$-valor $<0,01$ ) que la prevalencia de $0,7 \%$ reportada (año 2016) para la Región Metropolitana (RM); sugerimos que es consecuencia de la implementación del programa nacional y local. La transmisión congénita de $0 \%$ es acorde a la tasa de [0,91 x 10.000] reportada el 2017 para la RM. En base al aprendizaje adquirido, durante el desarrollo de la investigación, se entrega sugerencias para aportar al funcionamiento del programa.

Palabras clave: Trypanosoma cruzi; enfermedad de Chagas; transmisión congénita; prevalencia; Chile. up. In all, congenital transmission $(0 \%)$ was ruled out. Nifurtimox treatment of the mothers were completed in $30 \%$ of them. It is concluded that the implementation of the program in the HDFBC fulfills the objectives of screening, detection and diagnosis of CHD, so as the timely follow-up and pharmacologic treatment of the newborn. The $0.085 \%$ prevalence of CHD in pregnant women as the $0.20 \%$ in total population are significantly lower ( $\mathrm{p}$-valor $<0.01$ ) than the $0.7 \%$ reported in 2016 for the Metropolitan Region (MR), a fact that we suggest is a consequence of the national program implementation. Likewise, the $0 \%$ transplacental transmission is accorded to the rate of $0.91 \times 10.000$ live births reported in 2017 for the MR. Based on the learning acquired during the development of the research, recommendations are given to contribute to the operation of the program.

Keywords: Trypanosoma cruzi; Chagas disease; congenital transmission; prevalence; Chile.

\section{Introducción}

L a enfermedad de Chagas (ECH) es una infección parasitaria, sistémica, crónica, potencialmente mortal, cuyo agente etiológico es el protozoo Trypanosoma cruzi. Hoy, continúa siendo un importante problema de salud pública, focalizándose en zonas endémicas de 21 países de la parte continental de América Latina; se estima en alrededor de 12.000 las muertes anuales, entre 8 y 11 millones los afectados y en 65 a 100 millones las personas en riesgo de infección. En la última década se ha observado con mayor frecuencia la ECH en Estados Unidos de América, Canadá, algunos países europeos y otros del Pacífico occidental, como consecuencia de la movilidad poblacional entre América Latina y el resto del mundo ${ }^{1,2,9}$.

La transmisión natural de $T$. cruzi, ocurre entre insectos hematófagos y animales mamíferos, involucrando ocasionalmente al ser humano. En Chile, Triatoma infestans (vinchuca), es el principal vector relacionado con la prevalencia de la $\mathrm{ECH}$, el que actualmente posee un hábitat peri-domiciliario. Existen otras formas de transmisión tales como transplacentaria, transfusional, oral y accidental en laboratorio. La ECH es una parasitosis marcada por inequidades sociales, pero además comprende reservorios domésticos y silvestres lo que hace imposible su erradicación. No obstante, es posible interrumpir los distintos mecanismos de transmisión hacia el ser humano ${ }^{2}$.

La Iniciativa "INCOSUR" medió la interrupción de la transmisión vectorial y transfusional en Uruguay, Chile y Brasil, así como en algunas zonas de Paraguay, Perú y Bolivia $^{3}$. Chile, en el año 1999 alcanzó la meta de reducción de los índices de infestación domiciliaria, logrando la certificación de la interrupción de la transmisión por la vía vectorial ${ }^{4}$. Hoy, Chile aspira a la certificación de la interrupción de la transmisión transfusional de $T$. cruzi, como consecuencia de la implementación del cribado obligatorio de la sangre donada en todo el territorio nacional ${ }^{2,3}$.

La Organización Mundial de la Salud estima, que en Latinoamérica existen alrededor de 1.200 .000 mujeres en edad fértil infectadas con T. cruzi. El 50\% se concentra en México, Argentina y Colombia, con frecuencias de transmisión transplacentaria que varían de 1 a $40 \%$, originando 15.000 nuevos casos de ECH por esta vía al año ${ }^{3,8}$. La tasa de transmisión congénita de $T$. cruzi se estima en $5 \%$ para los niños nacidos en zonas endémicas, con un rango entre 0 y $28,6 \%{ }^{5}$.

El tratamiento antiparasitario en niños tiene una efectividad cercana a $100 \%$, si se inicia durante el primer año de vida, y en adolescentes y adultos jóvenes disminuye la probabilidad de desarrollar futuras lesiones cardiovasculares o digestivas. Por ello, es clave la pesquisa precoz del parásito en mujeres gestantes, $\mathrm{RN}$ y lactantes, especialmente en zonas endémicas, con el fin de otorgar tratamiento farmacológico oportuno ${ }^{2,6,7}$.

La Encuesta Nacional de Salud (ENS) 2016-2017 reportó una prevalencia de $0,7 \%$ de $T$. cruzi en la Región Metropolitana. También, estimó en 120.000 a las personas infectadas, donde 12.000 serían mujeres en edad fértil. La frecuencia de transmisión de madre a hijo varía de 2,1 a $9,8 \%$, de quienes 70 a $80 \%$ nace asintomático y, en aquellos que se presentan manifestaciones clínicas, éstas semejan el síndrome de TORCH. La vía transplacentaria es el principal mecanismo de transmisión activa en Chile $^{8,9,13,18}$

El año 2014, el Gobierno instauró el Programa Nacional para la Prevención y Control de la ECH con la indicación de implementar el cribado obligatorio a toda mujer em- 


\section{Análisis serológico y RPC para detección de T. cruzi}

El cribado se realizó mediante técnica de inmunoquimioluminiscencia (Biokit Chagas-Werfen), que identifica anticuerpos IgM e IgG anti $T$. cruzi en muestras de plasma. El punto de corte es 0,90-1,00; así, una muestra reactiva implica un resultado mayor a $1,00 \mathrm{y}$ no reactiva menor a 0,90 . Las muestras reactivas se derivaron al centro de referencia nacional (ISP), para su confirmación, la que se obtiene mediante un algoritmo de técnicas complementarias de inmunofluorescencia indirecta (IFI), ELISA y/o WB. El plasma se conservó a $-20^{\circ} \mathrm{C}$. La transmisión congénita de $T$. cruzi se confirma por detección del genoma de $T$. cruzi en la muestra de sangre del RN y/o lactante, acorde a la Norma Técnica $2014^{2}$. La reacción de polimerasa en cadena (RPC) amplifica un fragmento de la región conservada del ADN minicírculo presente en el kinetoplasto del parásito. $\mathrm{La}$ mezcla de reacción contiene $10 \mathrm{mM}$ de cada partidor: L121-[AAATAATGTACGGGKGAGATGCATGA] y L122-[GGTTCGATTGGGGTTGGTGTAATATA], los que generan un producto de amplificación de $330 \mathrm{pb}$ kADN en presencia de Taq Platinum 5 U/uL, $200 \mathrm{mM}$ dNTPs y $3 \mathrm{mM} \mathrm{MgCl}_{2}$. Los ciclos de amplificación son: 3 minutos denaturación a $94{ }^{\circ} \mathrm{C} ; 2$ ciclos de 1 minuto a $97,5{ }^{\circ} \mathrm{C} ; 2$ minutos a $64{ }^{\circ} \mathrm{C}$ seguidos de 33 ciclos de 1 minuto a $94{ }^{\circ} \mathrm{C}, 1 \mathrm{~min}$ a $62{ }^{\circ} \mathrm{C}$ más una extensión final de 10 minutos a $72{ }^{\circ} \mathrm{C}^{10,18}$.

Diseño metodológico: Estudio observacional, retrospectivo, de la población cribada, y luego confirmada con ECH, durante el período 1 de abril de 2016 a 31 de julio de 2018. Los análisis se realizaron en la Sección Chagas del laboratorio clínico del HDFBC. Se determinó, la prevalencia de los anticuerpos anti $T$. cruzi en población total, mujeres gestantes y población no gestante junto a sus respectivas prevalencias de ECH. También, se observó la tasa de transmisión transplacentaria mediante el seguimiento de los RN y lactantes hijos de madres chagásicas hasta descartar la infección y se ofreció tratamiento antiparasitario a las madres que continuaron controles y a todos los pacientes infectados.

\section{Tratamiento}

Las mujeres gestantes se trataron con nifurtimox en dosis de $600 \mathrm{mg} / \mathrm{d}$ ía [7-10 mg/kg]; considerando la tolerancia individual, según lo recomendado en la Guía Clínica del Ministerio de Salud de Chile 7 . Se solicitó consentimiento informado. El tratamiento lo realizó un médico parasitólogo adscrito al policlínico de Chagas del HDFBC.

\section{Consideraciones éticas}

El protocolo diseñado por los investigadores del HDFBC propuso analizar las bases de datos que contenían 
los resultados e información obtenida en el laboratorio clínico luego del cribado y la confirmación diagnóstica de $\mathrm{ECH}$ en mujeres gestantes, niños y adultos, junto a algunos datos del tratamiento farmacológico. La información recopilada se trató como datos anónimos. El estudio no requirió de consentimiento informado de los pacientes, ya que sólo se analizan los datos epidemiológicos obtenidos en el período. El protocolo fue aprobado por el Comité de Ética Científica del HDFBC según acta de resolución interna $\mathrm{N}^{\circ} 06 / 2019$.

\section{Análisis estadístico}

Para determinar el efecto de la implementación del programa sobre la prevalencia de la ECH se utilizó la prueba de hipótesis para la comparación de dos muestras para proporción. Se testeó la proporción obtenida de 5.000 pacientes contenida en el "Informe Programa Nacional Integral de Enfermedad de Chagas, 2016 ${ }^{12}$ y del "Informe

\begin{tabular}{|c|c|c|c|c|c|}
\hline Comuna & Establecimiento & $\begin{array}{c}\text { Período at } \\
\mathbf{n} \\
\text { reactivos }^{\#}\end{array}$ & $\begin{array}{c}\text { bril de } 2016 \text { a } \\
\text { Confirmado } \\
(+)^{5}\end{array}$ & $\begin{array}{c}\text { julio de } 2018 \\
\text { Confirmado } \\
(-)^{5}\end{array}$ & $\begin{array}{c}\text { Casos } \\
\text { ECH }\end{array}$ \\
\hline \multirow[t]{3}{*}{ Quinta Normal } & CESFAM Andes & 0 & 0 & 0 & \\
\hline & CESFAM Garín & 2 & 0 & 2 & 0 \\
\hline & CESFAM Lo Franco & 2 & 0 & 2 & \\
\hline \multirow[t]{5}{*}{ Cerro Navia } & CESFAM Cerro Navia & 4 & 2 & 2 & \\
\hline & CESFAM Steeger & 5 & 0 & 5 & \\
\hline & CESFAM Lo Amor & 5 & 2 & 3 & 5 \\
\hline & CESFAM Albertz & 1 & 0 & 1 & \\
\hline & CECOSF Los Lagos & 1 & 1 & 0 & \\
\hline \multirow[t]{4}{*}{ Renca } & CESFAM Hernán Urzúa & 3 & 1 & 2 & \\
\hline & CESFAM Bicentenario & 1 & 1 & 0 & \\
\hline & Centro Salud Renca & 3 & 1 & 2 & 6 \\
\hline & Huamachuco & 5 & 3 & 2 & \\
\hline Padre Hurtado & CESFAM Juan Pablo II & 4 & 1 & 3 & 1 \\
\hline \multirow[t]{2}{*}{ Peñaflor } & CESFAM Monckeberg & 4 & 2 & 2 & \\
\hline & CESFAM Peñaflor & 3 & 0 & 3 & 2 \\
\hline \multirow[t]{7}{*}{ HDFBC } & Poli Gastro & 1 & 1 & 0 & \\
\hline & Parto & 4 & 2 & 2 & \\
\hline & Gine & 2 & 0 & 2 & \\
\hline & Poli Medicina & 6 & 6 & 0 & 14 \\
\hline & CAAE Providencia & 2 & 2 & 0 & \\
\hline & Urgencia ARO & 3 & 2 & 1 & \\
\hline & Poli FAR & 1 & 1 & 0 & \\
\hline Total & & 62 & 28 & 34 & 28 \\
\hline
\end{tabular}

Indicadores Programáticos Plan Nacional Enfermedad de Chagas-2016 ${ }^{13}$, en los que se reportó una prevalencia de $0,7 \%$. Por su parte, en la presente investigación se determinó una prevalencia de $0,085 \%$ para la población gestante estudiada obtenida de una muestra de 11.780 personas y de una prevalencia de $0,2 \%$ en la población total atendida igual a 14.012 sujetos. Las prevalencias reportadas en esta investigación son significativamente menores ( $\mathrm{p}$-valor $<0,01)$ a la cifra de referencia publicada igual a $0,7 \%$. Todos los análisis se realizaron con los softwares estadístico R-Studio y con EpiInfo.

\section{Resultados}

En el laboratorio clínico del HDFBC se estudió a un total de 14.012 muestras de pacientes. Éstos provinieron de 15 establecimientos APS (CESFAM, CESCOF) pertenecientes a seis comunas (Quinta Normal, Cerro Navia, Renca, Padre Hurtado, Talagante, Peñaflor) y del HDFBC, todos adscritos al SSMOCC (Tabla 1); las muestras fueron recibidas entre julio de 2016 y julio de 2018 . De la población total, 62 pacientes resultaron reactivos para la prueba de anticuerpos anti $T$. cruzi $(0,53 \%$ seropositividad). De ellos, $34(54,8 \%)$ se confirmaron como negativos (falsos positivos) y $28(45,2 \%)$ se confirmaron positivos (Tabla 1). Los casos positivos correspondían a los centros de atención primaria de Cerro Navia (17,8\%), Renca $(21,4 \%)$, Padre Hurtado (3,5\%), Peñaflor $(7,1 \%)$ y al HDFBC (50,0\%), a excepción de Quinta Normal $(0 \%)$ (Tabla 2).

Así, la prevalencia de la ECH fue de $0,2 \%$ en la población total (Tabla 3 ). En cuanto a la población gestante (11.780), 41 muestras fueron reactivas; 10 $(24,4 \%)$ mujeres embarazadas se confirmaron con ECH y $31(75,6 \%)$ como negativas, de modo que la prevalencia estimada fue de $0,085 \%$ (Tabla 4). También, se recibió a pacientes no gestantes, beneficiarios del programa,

\begin{tabular}{lcc|}
$\begin{array}{l}\text { Tabla 2. Distribución geográfica y porcentual de los casos } \\
\text { de ECH }\end{array}$ & Casos ECH & $\%$ Casos \\
\hline Comuna & 0 & 0,00 \\
\hline Quinta Normal & 5 & 17,85 \\
\hline Cerro Navia & 6 & 21,42 \\
\hline Renca & 1 & 3,57 \\
\hline Padre Hurtado & 2 & 7,14 \\
\hline Peñaflor & 14 & 50,0 \\
\hline HDFBC & 28 & 100 \\
\hline Total & & \\
\hline
\end{tabular}


con sospecha clínica de la enfermedad y a estudios de contacto de casos con ECH. Tal población fue de 2.232; de éstas, 21 muestras resultaron reactivas; 18 (85,7\%) se confirmaron con ECH y $3(14,2 \%)$ como negativas. En este caso, la prevalencia observada fue de $0,81 \%$ (Tabla 5). Los resultados se presentan resumidos en la Figura 1. Cabe mencionar, que la confirmación serológica de cada paciente reactivo para anticuerpos anti $T$. cruzi se llevó a cabo en el ISP, actual centro de referencia nacional.

\section{Enfermedad de Chagas y embarazo}

La distribución de nacionalidad entre las 10 mujeres gestantes chagásicas fue de $8(80 \%)$ chilenas y $2(20 \%)$ bolivianas. La edad media fue de 29 años, con rango de 37 a 19 años. Ninguna presentó síntomas característicos de ECH aguda o crónica ni reportó haber recibido transfusiones sanguíneas.

\section{Hijos de madres chagásicas}

Se estudió $11 \mathrm{RN}$ y/o lactantes. Uno de éstos, hijo de madre chagásica controlada en otro centro, lamentablemente falleció por otra causa dentro del primer mes de vida. En los otros 10 casos no se detectó ADN de T. cruzi en ninguna de las muestras seriadas de sangre (Tabla 6) que corresponde analizar hasta los 9 meses de vida, según lo indica la norma elaborada por MINSAl. En paralelo,

\begin{tabular}{|ccccc|}
\hline \multicolumn{4}{|l|}{ Tabla 3. Prevalencia de la ECH en la población total cribada } \\
\hline $\mathbf{N}^{\circ}$ tamizado & Reactivos & Negativos & Positivos & Prevalencia \% \\
14.012 & 62 & $34(54,8 \%)$ & $28(45,2 \%)$ & 0,2 \\
\hline
\end{tabular}

\begin{tabular}{|ccccc|}
\hline \multicolumn{4}{|c|}{ Tabla 4. Prevalencia de la enfermedad de Chagas en población gestante } \\
\hline $\mathbf{N}^{\circ}$ tamizado & Reactivos & Negativos & Positivos & Prevalencia $\%$ \\
11.780 & 41 & $31(75,6 \%)$ & $10(34,4 \%)$ & 0,085 \\
\hline
\end{tabular}

\begin{tabular}{|ccccc|}
\hline \multicolumn{5}{|c|}{ Tabla 5. Prevalencia de la ECH en población no gestante } \\
\hline $\mathbf{N}^{\circ}$ tamizado & Reactivos & Negativos & Positivos & Prevalencia \% \\
2.232 & 21 & $3(14,2 \%)$ & $18(85,7 \%)$ & 0,81 \\
\hline
\end{tabular}

al análisis de RPC, el ISP realizó ensayos IFI titulado y ELISA. Posterior a los nueve meses de vida la prueba serológica de elección fue la inmunoquimioluminiscencia, que después de un resultado no reactivo (Tabla 6) permitió otorgar el alta. Cabe mencionar, que el seguimiento

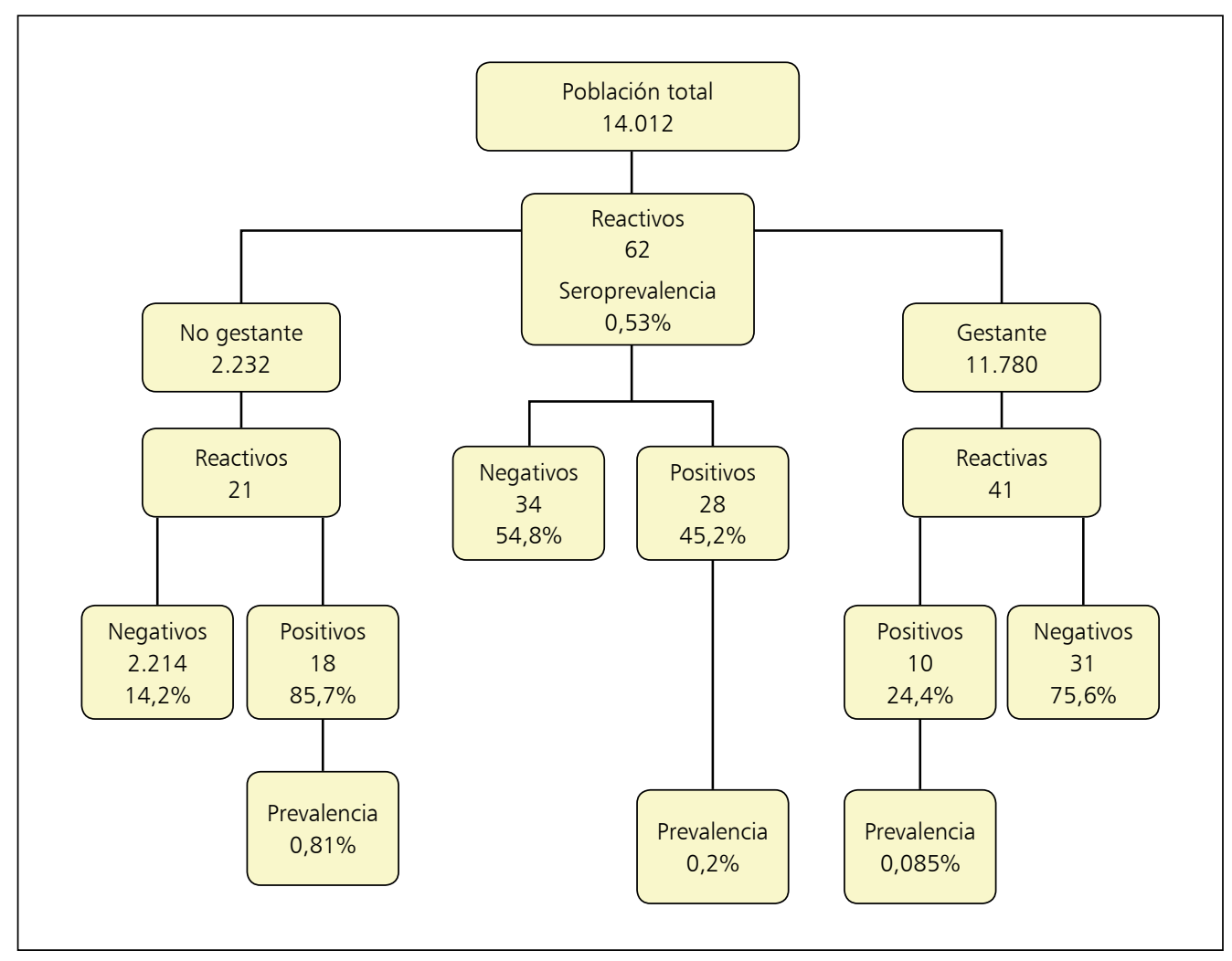

Figura 1. Determinación de la prevalencia de la enfermedad de Chagas en población gestante, no gestante y total atendida en el HDFBC. La población total (14.012) estudiada presenta una seroprevalencia de $0,53 \%$ y una prevalencia de la ECH de $0,2 \%$; en tanto que en las mujeres gestantes (11.780) 10 embarazadas se confirmaron positivas calculándose la prevalencia en $0,085 \%$. En el grupo no gestante (2.232) se confirmó a 18 pacientes con ECH resultando en una prevalencia de $0,81 \%$. 


\begin{tabular}{|c|c|c|c|c|c|c|c|}
\hline \multirow[b]{2}{*}{ Paciente } & \multirow[b]{2}{*}{ Muestra } & \multicolumn{3}{|c|}{ ISP-centro referencia nacional } & \multicolumn{2}{|l|}{ HDFBC } & \multirow[b]{2}{*}{ Observación } \\
\hline & & RPC anti $T$. cruzi & $\mathrm{IFI} / \mathrm{tit}$ & ELISA & CLIA & Edad & \\
\hline \multirow[t]{4}{*}{11} & 1 & No detectable & Pos/1/40 & Positivo & NA & $2 \mathrm{~m}$ & Seguimiento \\
\hline & 2 & No detectable & Negativo & Negativo & NA & $4 \mathrm{~m}$ & Seguimiento \\
\hline & 3 & No detectable & Negativo & Negativo & NA & 1a $1 \mathrm{~m}$ & Seguimiento \\
\hline & 4 & NA & NA & NA & No Reactivo & $1 \mathrm{a} 4 \mathrm{~m}$ & Alta \\
\hline \multirow[t]{3}{*}{12} & 1 & No detectable & Pos $/ 1 / 80$ & Positivo & NA & $1 \mathrm{~m}$ & Seguimiento \\
\hline & 2 & No detectable & Pos $/ 1 / 80$ & Positivo & NA & $2 \mathrm{~m}$ & Seguimiento \\
\hline & 3 & NA & NA & NA & No Reactivo & $1 \mathrm{a} 6 \mathrm{~m}$ & Alta \\
\hline \multirow[t]{2}{*}{13} & 1 & No detectable & Pos $/ 1 / 80$ & Positivo & NA & $4 \mathrm{~m}$ & Seguimiento \\
\hline & 2 & NA & & & No Reactivo & 1a $2 m$ & Alta \\
\hline \multirow[t]{3}{*}{14} & 1 & No detectable & Pos/1/160 & Positivo & NA & $1 \mathrm{~m}$ & Seguimiento \\
\hline & 2 & NA & NA & NA & No Reactivo & $9 \mathrm{~m}$ & Seguimiento \\
\hline & 3 & NA & NA & NA & No Reactivo & 1a $1 \mathrm{~m}$ & Alta \\
\hline \multirow[t]{3}{*}{15} & 1 & No detectable & Pos/1/320 & Positivo & NA & RN & Seguimiento \\
\hline & 2 & NA & NA & NA & No Reactivo & $9 \mathrm{~m}$ & Seguimiento \\
\hline & 3 & NA & NA & NA & No Reactivo & $10 \mathrm{~m}$ & Alta \\
\hline \multirow[t]{5}{*}{16} & 1 & No detectable & Pos/1/160 & Positivo & NA & RN & Seguimiento \\
\hline & 2 & No detectable & Por/1/80 & Positivo & NA & $2 \mathrm{~m}$ & Seguimiento \\
\hline & 3 & No detectable & Negativo & Positivo & NA & $10 \mathrm{~m}$ & Seguimiento \\
\hline & 4 & NA & NA & NA & No Reactivo & $1 \mathrm{a} 6 \mathrm{~m}$ & Seguimiento \\
\hline & 5 & NA & NA & NA & No Reactivo & 1a $10 \mathrm{~m}$ & Alta \\
\hline \multirow[t]{2}{*}{17} & 1 & No detectable & Pos/1/160 & Positivo & NA & RN & Seguimiento \\
\hline & 2 & NA & NA & NA & No Reactivo & $1 \mathrm{a} 7 \mathrm{~m}$ & Alta \\
\hline \multirow[t]{3}{*}{18} & 1 & No detectable & Pos/1/80 & Positivo & NA & RN & Seguimiento \\
\hline & 2 & No detectable & Pos/1/20 & Positivo & NA & $2 \mathrm{~m}$ & Seguimiento \\
\hline & 3 & No detectable & Negativo & Negativo & NA & $9 \mathrm{~m}$ & Seguimiento \\
\hline \multirow[t]{2}{*}{19} & 1 & No detectable & Pos/1/160 & Positivo & NA & RN & Seguimiento \\
\hline & 2 & NA & NA & NA & No Reactivo & $11 \mathrm{~m}$ & Seguimiento \\
\hline \multirow[t]{3}{*}{20} & 1 & No detectable & Pos/1/80 & Positivo & NA & RN & Seguimiento \\
\hline & 2 & NA & NA & NA & No Reactivo & $10 \mathrm{~m}$ & Seguimiento \\
\hline & 3 & NA & NA & NA & No Reactivo & 1a $2 m$ & Alta \\
\hline
\end{tabular}

de cada RN y lactante, que se presentan en la Tabla 6, corresponden a datos actualizados al año 2020.

El análisis estadístico de comparación de dos muestras para proporción demostró una prevalencia de $0,2 \%$ para la población total de muestras del HDFBC. En tanto que la prevalencia de la ECH en la población gestante fue de $0,085 \%$ y en la no gestante de $0,81 \%$. La transmisión transplacentaria observada fue de $0 \%$.

\section{Tratamiento anti-parasitario}

De las 10 mujeres embarazadas chagásicas sólo 30\% había concluido el tratamiento con nifurtimox. Llama la atención que $70 \%$ no asista a los controles de la especialidad y postergue su tratamiento y que tampoco cumpla con los controles de seguimiento de su hijo, motivo por el que se solicitó apoyo a la atención primaria para cumplir con el seguimiento (Tabla 7). 


\section{Discusión y conclusiones}

Nuestra investigación demostró que la prevalencia de la ECH es de $0,2 \%$ en la población total estudiada, significativamente menor ( $\mathrm{p}$-valor $<0,01$ ) que la prevalencia de $0,7 \%$ reportada con anterioridad para la Región Metropolitana en la ENS 2009-2010 y la ENS 2016-2017'2,13. En la misma fuente, se informa una prevalencia de $1,5 \%$ para zona rural y de $0,6 \%$ para zona urbana ${ }^{12}$. Así, la presente investigación sugiere que nuestra población asignada, dependiente SSMOCC, presenta una prevalencia acorde a la descrita para Chile para zonas de endemia intermedia, en rango de 1,5 a 3,5 x 100.000 habitantes, según se cita en informes oficiales emitidos recientemente ${ }^{13,20}$.

La población gestante, objeto de estudio de nuestra investigación, presentó una prevalencia de 0,085\%. Lamentablemente, en la literatura científica reciente no se ha publicado estudios de prevalencia para mujeres embarazadas de la Región Metropolita (SSMOCC). De hecho, para la presente investigación se realizó una búsqueda en PubMed con las palabras clave "Chagas, Chile", lo que generó 584 referencias de las cuales en sólo tres (2017, 2013 y 2010) se citaban datos de prevalencia de la ECH en el país. Al filtrar la búsqueda a cinco años resultaron 158 referencias, las mismas tres anteriores y de nuestro interés ${ }^{9,14,18}$. En el repositorio Scielo, la búsqueda "Chagas, Chile” generó 62 referencias, dos citaban prevalencia de ECH en coincidencia con PubMed. De esta manera, la información publicada respecto a la prevalencia de la ECH en mujeres gestantes se redujo a la Provincia de Choapa, donde se reportó hace 10 años como de 3,4\%. Actualmente, Choapa permanece como zona de alta endemia, rango [1,9 a 6,2 x 100.000 habitantes $]^{14}$.

En la presente investigación no observamos casos de transmisión transplacentaria $(0 \%)$ de $T$. cruzi, valor que requiere validación a través del seguimiento de una población gestante representativa, debido a que nuestro grupo de estudio estuvo conformado por sólo 10 madres chagásicas. Cabe mencionar, que el informe MINSAL 2017 reportó una tasa de transmisión congénita para el país de 0,91 x 10.000 nacidos vivo ${ }^{20}$ y la $\mathrm{ENS}^{12,13}$ una frecuencia de transmisión de [2,1-9,8\%]. Asimismo, que la tasa de incidencia de los casos congénitos en mujeres embarazadas con cribado para ECH es de $0,26 \%{ }^{20}$. No obstante, en los informes programáticos del MINSAL no se encontraron datos acerca de la transmisión congénita en el SSMOCC, contrariamente a lo que ocurrió para otros servicios de salud de la Región Metropolitana ${ }^{12,13,20}$. Como información referencial, queremos citar a dos investigaciones conducidas hace 10 años en las regiones de Atacama y Valparaíso ( $\mathrm{IV}^{\circ}$ y $\mathrm{V}^{\circ}$ regiones), ambas de alta endemia. La primera, de Jercic y cols., (2010), describió una tasa de transmisión congénita de $8,4 \%$ aplicando la técnica de RPC para la detección del ADN de T. cruzi en

\begin{tabular}{|ccc|}
\hline \multicolumn{2}{|c|}{ Tabla 7. Tratamiento de mujeres gestantes con enfermedad de Chagas } \\
\hline Paciente & Nifurtimox\% & Observación \\
1 & Pendiente & Descontinúa controles \\
2 & Pendiente & Descontinúa controles \\
3 & Pendiente & Descontinúa controles \\
4 & Pendiente & Descontinúa controles \\
5 & Pendiente & Descontinúa controles \\
6 & Terminado & Completó tratamiento \\
7 & Pendiente & Descontinúa controles \\
8 & Terminado & Tratada previo embarazo post-aborto \\
9 & Pendiente & Descontinúa controles \\
10 & Terminado & Completó tratamiento \\
\hline \%Dosis de $600 \mathrm{mg} /$ día $\left[7-10\right.$ mg/kg]; según tolerancia. ${ }^{\text {*Providencia. }}$ \\
\hline
\end{tabular}

sangre de cordón umbilical ${ }^{18}$. La segunda, que se realizó en forma simultánea a la anterior, reportó una frecuencia de transmisión transplacentaria de $4,7 \%$ para la provincia de Choapa utilizando la técnica serológica ELISA ${ }^{14}$. Considerando el valor global promedio de $5 \%$, aceptado para la transmisión congénita en áreas endémicas de latinoamericanas ${ }^{6}$, o los valores recientemente publicados de 6,6 y $6,3 \%$ para Argentina y México, respectivamente, para el período 2011-2014 ${ }^{15}$, podemos especular que en la Región Metropolitana la tasa de transmisión transplacentaria debería ser menor a la reportada hace diez años para zonas con alta endemia, gracias a la implementación de programas nacionales para el control y prevención de la enfermedad de Chagas ${ }^{19,20}$.

Para lograr el control de la transmisión transplacentaria en el país, también se debe considerar la existencia de factores relevantes para la evolución de la infección, tales como el tipo de parásito infectante, grado de parasitemia de la madre y competencia del sistema inmune, junto a la interacción entre el parásito y la placenta (primera barrera fetal) y del propio sistema inmune del feto ${ }^{5}$. Una posible explicación a la baja incidencia de $\mathrm{RN}$ infectados puede ser las mejoras en el estado nutricional de las mujeres gestantes y el buen estado de su sistema inmunológico. De hecho, la transmisión vertical puede estar asociada a la deficiencia de la inmunidad natural y/o adquirida, situación que disminuye la producción de IFN- $\gamma$ en respuesta a T. cruzi, hecho clave para la activación de los macrófagos y la eliminación del parásito. Recientemente, también se ha comenzado a atribuir a la microbiota intestinal materna cierto control sobre la transmisión congénita de T. cruzi $i^{5}$. Más aún, algunos estudios indican que la transmisión vertical puede ser prevenida cuando la mujer infectada recibe tratamiento pre-concepcional ${ }^{6,17}$. 
En base a los resultados de nuestra investigación podemos concluir, que la implementación del Programa de Control y Prevención de la Enfermedad de Chagas en el HDFBC ha cumplido con los objetivos de detección y diagnóstico de la ECH. Ha mediado el cribado de la población gestante, así como la pesquisa precoz y seguimiento del RN infectado, hasta descartar o confirmar la enfermedad. De igual manera, ofrece diagnóstico a los grupos de riesgo y ofrece la oportunidad de tratamiento antiparasitario a los infectados (familiares contactos de casos positivos y pacientes con sospecha clínica de la enfermedad). Por otra parte, nuestra investigación propone que la prevalencia de la $\mathrm{ECH}$, en nuestra población asignada del SSMOCC, corresponde al rango de zona geográfica de endemia intermedia.

De igual manera, consideramos que nuestro estudio da a conocer cifras de prevalencia actualizadas y referidas específicamente a nuestra población gestante, hecho que constituye un aporte de nueva evidencia científica.Nuestro aprendizaje, durante la implementación y desarrollo del programa, ha favorecido la identificación de dificultades que nos permite entregar sugerencias que aporten al mejor desarrollo y eficacia del programa, a través de ideas de innovación para el control de la transmisión transplacentaria en el país. Se propone considerar la implementación de estrategias complementarias tales como: mejorar el diagnóstico precoz del RN a través de la implementación de técnicas moleculares en formato point-of-care ${ }^{16}$; implementar el cribado de mujeres en edad fértil, al menos una vez en la vida, de modo de pesquisar la ECH previo al embarazo ${ }^{17}$; coordinar el seguimiento del RN en colaboración con el control sano de la atención primaria, de manera de establecer una estrategia de identificación de aquellos lactantes que no han completado su seguimiento. La postergación reiterada, observada en nuestro estudio, aparentemente obedecería a dificultades de movilización desde sus lugares de residencia a la sede del HDFBC, provisoriamente ubicada en la remota comuna de Providencia; se espera que ello mejore con el traslado de éste a sus nuevas instalaciones, ubicadas en Cerro Navia. Por lo mismo, educar a la población gestante y mujeres en edad fértil, ya que la pérdida de la percepción del riesgo a $T$. cruzi es una amenaza para el control y prevención de la ECH. Tal hecho se infiere del observar que la mortalidad de la tripanosomiasis en Chile no ha presentado una variación favorable en 65 años. Sin embargo, la médico parasitóloga del programa tiene la percepción de que, transcurridos varios años desde el inicio del programa, las pacientes chagásicas más recientes muestran adhesión más alta a los controles, evidenciando mayor compromiso con el control de su enfermedad, aparentemente debido al acceso temprano a la educación de un estado de portador de T. cruzi $^{9}$. Si la ECH no es tratada oportunamente, la parasitosis persiste toda la vida provocando un riesgo vital potencial. Por tal motivo, es clave abordar este desafío de salud pública de forma integral, involucrando a los distintos actores sociales, tanto nacionales como internacionales, con el fin de lograr los objetivos sanitarios del país.

Agradecimientos. A la Sección Parasitología, Centro de Referencia Nacional para la Enfermedad de Chagas, del Instituto de Salud Pública de Chile, quienes realizaron la confirmación diagnóstica de la enfermedad, según normativa nacional. Asimismo, a Jasna Barahona por la transcripción de los datos de nacionalidad y tratamiento de los diez casos confirmados con enfermedad de Chagas.

\section{Referencias bibliográficas}

1.- Organización Mundial de la Salud (OMS). La enfermedad de Chagas (tripanosomiasis americana) 17 de abril de 2019 [Internet]. Disponible en: http://www.who.int/es/ news-room/fact-sheets/detail/chagas-disease(american-trypanosomiasis).

2.- Ministerio de Salud de Chile (MINSAL). Norma general técnica control y prevención nacional de la enfermedad de Chagas, febrero de 2014. [Internet]. Disponible en: https:// diprece.minsal.cl/wrdprss_minsal/wp-content/ uploads/2016/03/NORMA-TECNICA CHAGAS edici\%C3\%B3n-definitiva-140514. pdf.

3.- Organización Panamericana de Salud (OPS). El control de la Enfermedad de Chagas en los países del Cono Sur de América, 2002
[Internet]. Disponible en: http://www1.paho. org/spanish/ad/dpc/cd/dch-historia-incosur.pdf

4.- Pan American Health Organization. Southern Cone Initiative to Control/Eliminate Chagas Disease (INCOSUR), 2012 [Internet]. Disponible en: https://www.paho.org/hq/ index.php?option $=$ com_content\&view $=\mathrm{a}$ rticle\&id=6001:2011-iniciativa-cono-surcontrolar-eliminar-enfermedad-chagasincosur\&Itemid $=4219 \&$ lang $=$ es.

5.- Ceballos-Pomares J C, Cuéllar-Rufino S, Vázquez-Ortega M F, López-Domínguez J, Romero-Cruz V, Calderón-Garcidueñas A L. Inmunología de la enfermedad de Chagas congénita. Perinatol Reprod Hum. 2017; 31 (3): 144-50. https://doi.org/10.1016/j. rprh.2018.01.001.

6.- Carlier Y, Sosa-Estani S, Luquetti AO, Buekens P. Congenital Chagas disease: an update. Mem Inst Oswaldo Cruz. [Internet] 2015; 110 (3): 363-8. doi: 10.1590/007402760140405 .

7.- Ministerio de Salud de Chile (MISAL). Guía de Diagnóstico, Tratamiento y Prevención de la Enfermedad de Chagas [Internet] agosto de 2010. Disponible en: https://www. paho.org/panaftosa/index.php?option=com docman\&view=download\&category _ slug=zoonosis-779\&alias=207-guiaenfermedad-chagas- $7 \&$ Itemid $=518$.

8.- Organización Panamericana de la Salud (OPS). Enfermedad de Chagas en las Américas: una revisión de la situación actual de salud pública y su visión para el futuro. Washington D.C., 3 y 4 mayo 2018 [Internet]. Disponible en: https:// www.paho.org/hq/index.php?option=com docman\&view=download\&category slug=informes- tecnicos-6200\&alias $=45142$ - 
cl/wp- content/uploads/2016/07/Informe Situacion_Vigilancia_Ech_chile.pdf.

enfermedad-chagas-americas-una-revisionsituacion-actual-salud-publica- su-visionfuturo-informe-conclusiones-recomendaciones2018-142\&Itemid=270\&lang=en .

9.- Canals M, González C, Canals L, Canals A, Cáceres D, Alvarado S, et al. ¿Qué dicen los números de la evolución temporal de la enfermedad de Chagas? Rev Chil Infectol. 2017; 34 (2): 120-7.

10.- Schijman A G, Bisio M, Orellana L, Sued M, Duffy T, Mejía Jaramillo A M, Cura C, et al. International study to evaluate PCR methods for detection of Trypanosoma cruzi DNA in blood samples from Chagas Disease patients [Internet]. PLoS Negl Trop Dis. 2011; 5 (1): e931. doi: 10.1371/journal.pntd.0000931.

11.- Servicio de Salud Metropolitano Occidente de Santiago de Chile (SSMOCC). Ordinario Interno $\mathrm{N}^{\circ} 43$ 07/06/2016: Inicio establecimientos del Programa de Control y Prevención de la Enfermedad de Chagas. Subdirección de Gestión Asistencial.

12.- Ministerio de Salud de Chile (MINSAL). Informe Programa Nacional Integral de Enfermedad de Chagas. Julio de 2016 [Internet]. Disponible en: http://epi.minsal.
13.- Ministerio de Salud de Chile (MINSAL). Informe Indicadores Programáticos Plan Nacional Enfermedad de Chagas. 2016 [Internet]. Disponible en: https://diprece. minsal.cl/wrdprss_minsal/wp-content/ uploads/2017/06/ENFERMEDAD-DECHAGAS- 2016_01.pdf.

14.- Apt W, Zulantay I, Arnello M, Oddó D, González S, Rodríguez J, et al. Congenital infection by Trypanosoma cruzi in an endemic area of Chile: a multidisciplinary study. Trans $\mathrm{R}$ Soc Trop Med Hyg. 2013; 107 (2): 98-104. doi: 10.1093/trstmh/trs013.

15.- Buekens P, Cafferata M L, Alger J, Althabe F, Belizán J M, Bustamante N, et al. Congenital transmission of Trypanosoma cruzi in Argentina, Honduras, and Mexico: An observational prospective study. Am J Trop Med Hyg. 2018; 98 (2): 478-85. doi: 10.4269/ ajtmh.17-0516.

16.- Picado A, Cruz I, Redard-Jacot M, Schijman A G, Torrico F, Sosa-Estani S, et al. The burden of congenital Chagas disease and implementation of molecular diagnostic tools in Latin America.
BMJ Glob Health 2018; Oct 11; 3 (5): e001069. doi:10.1136/ bmjgh-2018-001069.

17.- Fabbro D L, Danesi E, Olivera V, Codebó M O, Denner S, Heredia C, et al. Trypanocide treatment of women infected with Trypanosoma cruzi and its effect on preventing congenital Chagas. PLoS Negl Trop Dis. 2014; 8 (11): e3312. doi:10.1371/journal.pntd.0003312.

18.- Jercic M I, Mercado R, Villarroel R. Congenital Trypanosoma cruzi infection in neonates and infants from two regions of Chile where Chagas' disease is endemic. J Clin Microbiol 2010; 48 (10): 3824-6. doi: 10.1128/ JCM.00048-10.

19.- Ministerio de Salud-DIPLAS. Césped A. Situación epidem inmunoquimioluminiscencia biológica de la enfermedad de Chagas; 2018 [Internet] Disponible en: https://diprece. minsal.cl/wp-content/uploads/2018/11/ Situaci\%C3\%B3n-Epidemiologica-Nacional.Dr.-Adolfo-Cesped.pdf

20.- Informe Estrategia Integrada de Prevención y Control de la Enfermedad de Chagas 2017.pdf. [internet] Disponible en: https://diprece.minsal. cl/wp-content/uploads/2018/10/FOLLETO_ PROGRAMA-CHAGAS. 